\title{
Des jeux et des sociétés
}

Games and societies

\section{Gilles Brougère}

\section{(2) OpenEdition}

\section{Journals}

\section{Édition électronique}

URL : http://journals.openedition.org/sdj/2933

DOI : $10.4000 /$ sdj.2933

ISSN : 2269-2657

\section{Éditeur}

Laboratoire EXPERICE - Centre de Recherche Interuniversitaire Expérience Ressources Culturelles Education

\section{Référence électronique}

Gilles Brougère, «Des jeux et des sociétés », Sciences du jeu [En ligne], 14 | 2021, mis en ligne le 18 mars 2021, consulté le 06 avril 2021. URL : http://journals.openedition.org/sdj/2933 ; DOI : https:// doi.org/10.4000/sdj.2933

Ce document a été généré automatiquement le 6 avril 2021.

\section{c) (i) $(9)$}

La revue Sciences du jeu est mise à disposition selon les termes de la Licence Creative Commons Attribution - Pas d'Utilisation Commerciale - Pas de Modification 4.0 International. 


\title{
Des jeux et des sociétés
}

\author{
Games and societies
}

Gilles Brougère

\section{Présentation du texte}

1 Le texte qui suit date de 1979 et est le premier texte que j'ai publié avant même ma thèse de $3^{\mathrm{e}}$ cycle comme on disait à l'époque. Il a été écrit dans le cadre d'une recherche menée sous la direction de Robert Jaulin qui avait pour objet d'appliquer au monde du jeu et du jouet occidental les approches développées en ethnologie, en particulier en s'intéressant à la culture matérielle et aux techniques. Cette démarche que nous avions dénommée « ethnotechnologie » et qui donne son sous-titre à l'ouvrage (Jaulin, 1979) était loin d'être évidente et répandue à l'époque. Ce texte est donc marqué par la pensée de Jaulin soulignant "le vide» des pratiques occidentales au regard des sociétés, en particulier indiennes qu'il avait étudié et théorisé dans un ouvrage, Les chemins du vide, paru deux ans avant ce texte (Jaulin, 1977).

De nouveaux et récents lecteurs y ont trouvé des éléments pour penser le jeu de société contemporain, ce qui justifie la nouvelle publication de ce texte malgré certaines faiblesses et le fait qu'il évoque un état de l'édition des jeux de société bien éloigné de celui que l'on connaît aujourd'hui. Il semble cependant important de remettre dans leur contexte les quelques citations qui ont été retenues de ce texte, telles celles que l'on trouvera dans les différents articles de ce numéro.

3 Il constituait le premier texte d'un diptyque dont le second portait sur le jouet de la petite enfance (Brougère, 1979) et dont l'ensemble avait pour titre « Du jouet industriel au jouet rationalisé ", titre assez peu adéquat pour un texte dont la plus grande partie est consacrée au jeu de société, mais qui traduit le projet d'ensemble. Il s'agissait d'analyser la façon dont ces objets étaient conçus pour des destinataires potentiels, thème sur lequel je suis revenu bien des années après (Brougère et François, 2018). Cet aspect était pensé selon les modalités de la rationalisation, notion empruntée à 
Habermas (1973) et renvoyant à l'extension des domaines soumis à la décision rationnelle.

4 L'objectif était de comprendre le développement déjà conséquent dans les années 1960 et 1970 des jeux d'édition et plus particulièrement une sous-catégorie, ceux qui proposent des thèmes et non seulement des mécaniques ludiques, que j'ai appelés pour cette raison jeux thématiques. Le texte évoque un certain nombre de ces jeux et en dresse brièvement une liste, ce qui constitue le corpus de fait, et non rigoureusement constitué, sous-jacent à ce texte.

Ces jeux d'édition sont ceux que Bruce Whitehill (dans ce numéro) dénomme «jeux "propriétaires" - ceux dont les droits appartiennent à une société particulière » et dont son article montre le développement considérable aux Etats-Unis à partir du dernier tiers du $19^{\mathrm{e}}$ siècle, constituant une excellente illustration de ce que je montrais dans ce texte. Si cette question de propriété des droits est essentielle, la notion de jeu d'édition permet de mettre l'accent, au-delà des droits, sur le processus de conception, de production (d'édition au sens étroit du terme) et de distribution qui conduit à une transformation de la transmission de la culture ludique et du statut de l'objet. La propriété des droits en est une condition nécessaire. Whitehill (ce numéro) met également en évidence des aspects évoqués dans mon texte, le passage d'un objectif éducatif à une logique de divertissement et la reprise de thèmes qui viennent de la culture populaire et de l'actualité.

Dans le texte de 1979 je commence par mettre l'accent sur les divers modes de transmission de la règle ou du savoir-jouer ce qui continue à m'apparaître comme un élément essentiel. Pour traduire cela dans des catégories que j'ai développées ultérieurement, il s'agit de modalités différentes de la construction d'une culture ludique. Un autre aspect central de cette analyse est le lien entre jeu et manipulation d'un matériel, le statut de l'objet, de la culture matérielle qui rend possible à la fois l'action ludique et sa transmission.

7 Il ne faut pas oublier que l'analyse se rapporte à ce qu'offrait le marché des jeux d'édition à la fin des années 1970. Il s'est depuis transformé et nombre de remarques sur les jeux ne s'appliquent plus à la situation actuelle qui est marquée par la richesse des mécaniques ludiques associées à une thématique, ce qui n'était pas le cas au moment de l'écriture de ce texte (ou peut-être pas repéré par son auteur).

8 Une autre dimension de l'analyse renvoie à l'idée d'une transformation en profondeur de la société et la disparition des communautés qui pouvaient produire objets et jeux, une culture ludique étroitement communautaire. La confrontation à l'ethnologie et au travail de Robert Jaulin mettait en évidence cette dimension qui n'est sans doute plus présente dans mes travaux ultérieurs et dont on peut critiquer la vision par trop manichéenne.

9 Suit une analyse des modalités de jeu liées à l'offre dominante de la fin des années 1970, en mettant l'accent sur des jeux qui associent hasard et échange monétaire (ou leur équivalent) fictif. Cela conduisait à une nouvelle logique de jeu lié au matériel, mais aussi à l'importance prise par le hasard. Il s'agissait également d'une forme de recyclage du jeu d'argent. Par ailleurs est mis en évidence le fait que le jeu transforme et adapte à la situation ludique les éléments qui viennent de la réalité sociale visée. Ce qui importe est moins le réalisme que le fait que cela devienne jouable. Le texte se termine par une comparaison avec les descriptions de la théorie des jeux et les jeux d'entreprise, en soulignant le travail de modélisation, de simplification que l'on trouve 
dans ces divers dispositifs comme dans les jeux thématiques étudiés, mais dans ce dernier cas l'objectif en est le divertissement. Cependant, contrairement aux jeux d'entreprise, ce qui caractérise ces jeux est que l'on y joue des rôles éloignés de ceux que l'on tient au sein de la société. J'y soulignais comment ces jeux loin d'être des espaces d'apprentissage sont des espaces de divertissement où la référence sociale devenue thème n'est présente que pour l'amusement qu'elle peut produire.

Lié à ma double formation, philosophique et ethnologique, ma jeunesse m'a permis de proposer des théories sans trop m'assurer des preuves. Il convient de considérer qu'il s'agit là d'hypothèses, de cadres d'analyse ; à chacun de les utiliser s'ils lui paraissent utiles, de les réfuter ou de les ignorer. Relisant ce texte, et au vu de la faiblesse quantitative de la littérature sur les jeux de société, il ne m'a pas paru absurde de sortir ce texte de l'oubli, ce qu'avaient déjà fait, à ma grande surprise, certains chercheurs sur le jeu de société.

11 Le texte est reproduit tel qu'il a été publié, sans chercher à occulter ses faiblesses, avec uniquement de légères modifications pour corriger des fautes ou des rédactions peu claires, d'autant plus nécessaires que le texte a été publié alors que j'étais éloigné de Paris à une époque où aucun système ne permettait de corriger les épreuves à distance en dehors de la poste peu rapide en ce temps-là pour m'atteindre en Afrique où je vivais alors. Par ailleurs les références ont été adaptées aux règles de la revue. Les notes ajoutées dans cette édition portent la mention « note de 2021 », les autres sont celles de l'édition originale.

\section{Introduction}

Les nouveaux jeux de société (jeux d'édition, que nous appellerons jeux thématiques) se sont développés depuis le Monopoly et quelques autres (Deneuil, 1979). Il s'agit d'une part de tenter d'en dégager un sens en les mettant en rapport avec d'autres aspects de notre société auxquels ils se réfèrent plus ou moins explicitement, plus ou moins directement. D'autre part, nous verrons quelle continuité et quelles ruptures il peut y avoir entre eux et des jeux traditionnels. Les questions que nous posons de façon privilégiée et dont tout jeu ${ }^{1}$ doit relever au moins en partie sont relatives à leurs moyens de transmission (ou diffusion sociale), la règle, les thèmes évoqués et le rapport de ces thèmes à la règle.

La multiplicité de ces jeux et la complexité de notre société rendent impossible une analyse exhaustive. Tout au plus pouvons-nous tracer quelques chemins qui nous permettront de comprendre ce qu'il en est du jeu à règles aujourd'hui. Mais nous espérons pouvoir ainsi dégager des questions dont tout jeu doit plus ou moins relever, questions qui ne s'occupent jamais du jeu comme élément ou isolable, mais toujours inséré dans une société qui, seule, lui donne un sens. En effet, par la nécessité de réunir plusieurs joueurs et la référence commune nécessaire à des règles conventionnelles, le jeu de société a une liaison très étroite à la société où il s'insère. Il suppose donc pour exister une société qui maintient, ou diffuse ses règles. Il ne renvoie pas l'individu à luimême, à son propre imaginaire comme pourrait le faire un jeu spontané et irréfléchi, mais le suppose lié à un tissu social. En ce sens, il s'agit bien de jeu de société au plein sens du terme, jeu qui n'est possible que sur fond de société. Il importe de savoir si, audelà de cette évidence, le jeu réglé ne traduit pas une société dans la profondeur même de son fonctionnement, ou de celui qu'elle imagine être le sien. 
14 Dans les jeux que nous avons choisi d'analyser, nous verrons à la fois la simplicité formelle et une plus grande complexité, au moins apparente, au niveau des significations. Il s'agit d'ouvrir par là la voie vers l'analyse de ce qui a rendu possible dans nos sociétés de tels jeux.

\section{De la transmission des jeux}

Un des moyens de saisir la spécificité d'un jeu à règle est de voir de quelle façon il est disponible aux joueurs éventuels, c'est-à-dire à quelle diffusion sociale il fait appel. Etant donné des joueurs, par quel canal les règles leurs sont-elles connues ? Il s'agit là de ce qu'on peut appeler le rapport social immédiat au jeu. Différents types de transmission vont sans doute découler des rapports différenciés au jeu.

La transmission la plus traditionnelle est orale et visuelle, ou plus exactement elle se fait par apprentissage associé à l'audition de règles et à l'habitude de voir jouer. Que cette transmission soit codifiée, c'est-à-dire subordonnée à l'âge ou à tout autre caractère du futur joueur, ou qu'elle se fasse en dehors de toute régulation est peu important pour ce qui nous occupe ici. Il s'agit de remarquer que la société ou une partie de la société, une communauté déterminée, transmet parmi une multitude d'autres savoir-faire, un savoir-jouer qui est une possibilité de reconduire la communauté, une possibilité de permanence autour du maintien de certaines règles. Ceci peut se faire soit par l'insertion du nouveau joueur, soit par l'imitation de la part des enfants et adolescents du jeu adulte.

Aujourd'hui encore, la plupart des jeux de cartes, et surtout les plus populaires d'entre eux, ont une transmission de cette nature. Ainsi en est-il dans les régions où le jeu de belote est un jeu populaire se pratiquant dans les cafés (généralement entre hommes) sous la forme de communautés de joueurs relativement cohérentes: le lieu comme le temps du jeu y sont fortement déterminés. La transmission se fait pour les plus jeunes par le fait d'assister ou de participer aux parties, ce qui permet la permanence de la communauté au-delà des individus. Dans ce type de transmission, le matériel est simple et relativement uniforme, les jeux étant différenciés par les règles. C'est ainsi le cas d'un jeu de cartes acheté, mais polyvalent, lié au lieu du jeu (café) plus qu'à un joueur déterminé, ou des cailloux des jeux de calcul africains. Ces jeux ne supposent qu'un matériel partout disponible dans les sociétés qui les pratiquent. Par contre, ce matériel seul ne permet aucun jeu. Le jeu se situe donc essentiellement dans la communauté des joueurs qui possèdent la règle et non dans un matériel polyvalent qui n'induit de façon spécifique aucun jeu particulier. C'est la transmission de la règle, et non pas la possession du matériel, qui crée la communauté des joueurs qui peut être une partie de la société, ou celle-ci tout entière.

18 Il y a là autonomie complète de la communauté des joueurs qui n'a besoin d'aucune médiation extérieure pour jouer, et d'autant moins que dans les sociétés traditionnelles les jeux utilisent des éléments naturels (cailloux, grilles qu'il suffit de tracer sur le sable comme le Fanorona à Madagascar). Corrélativement le jeu s'éteint avec les joueurs, et la connaissance du jeu ne peut se faire que par celle des joueurs. De tels jeux peuvent se comparer à la littérature de transmission orale ainsi qu'aux transmissions du savoirfaire technique. 
Il existe une autre transmission qui, d'après les documents disponibles, se développa en Occident au plus tard à partir du XVII ${ }^{\mathrm{e}}$ siècle : il s'agit de recueils de règles tels que la Maison des jeux académiques de La Marinière $(1665)^{2}$ qui donne les règles de nombreux jeux de l'époque (Piquet, Oye, Tarot, tours de cartes, jeux de salon...). Sur ce modèle ont été édités de multiples Académies de jeux jusqu'à la fin du XIX ${ }^{\mathrm{e}}$ siècle. Et aujourd'hui encore, les éditeurs proposent des ouvrages sur le même modèle tels que les Livres de jeux et autres Codes des jeux. Il s'agit de présenter à des communautés potentielles de joueurs des règles (et uniquement des règles) qui leur permettent de jouer à partir, le plus souvent, d'un matériel simple, polyvalent et facilement disponible. Là encore la règle est première, et constitue le jeu.

Cependant sa possession n'est plus interne à une communauté, mais suppose la médiation externe d'un ouvrage, l'apprentissage de règles à partir d'une lecture, c'està-dire de façon abstraite. Aux joueurs la possibilité est donnée de choisir, une tradition n'imposant plus les jeux privilégiés qui perpétuent le groupe dans certaines de ses activités. Mais là où un choix sans détermination existe, il $\mathrm{y}$ a plus que rapport extérieur et contingent à ce qui en est l'objet.

1 Tout jeu pris parmi ceux qui sont offerts au choix du lecteur est bon pour constituer la communauté. Seul importe l'acte de jouer et non la forme privilégiée qu'il peut prendre au travers d'une tradition. Le jeu a quelque chose d'accidentel et non plus d'essentiel dans son rapport à la communauté. Lieu et temps perdent de leur importance au profit d'un jeu qui meuble du temps - le loisir - inemployé : d'où le recours, faute de tradition, à l'ouvrage, ou au savoir d'un joueur qui peut emprunter ses jeux à une autre communauté.

2 L'innovation de jeux (par l'auteur de l'ouvrage), la transmission des jeux encore réduits à une communauté limitée sont possibles et sont diffusées simultanément sur le même plan que les autres. La colonisation permit de diffuser des jeux de sociétés éloignées de façon massive et rapide, et non par la transmission selon un apprentissage. Pour le joueur apparaissent donc des jeux équivalents pour lesquels rien de particulier ne le détermine. L'écrit sépare le jeu de son insertion à une communauté et propose à toute communauté des jeux concurrents.

3 Cependant, il n'est transmis que des règles et il doit exister une communauté pour les recevoir, leur donner un sens. Les jeux que nous avons le projet d'étudier appartiennent à un autre type d'insertion sociale, la diffusion commerciale, qui n'est pas un caractère extérieur du jeu mais intrinsèquement lié à ce qu'il est. Il s'agit de jeux d'édition pourvus d'une part d'un « copyright ", et donc impossibles à copier, solidaires de leur forme commerciale. D'autre part, et c'est là leur caractère fondamental, leurs règles ne sont pas transmissibles isolément du matériel complexe dont elles donnent les conditions de manipulation. C'est-à-dire qu'il n'est pas possible de diffuser les règles hors le matériel, même par l'écrit. Il s'agit bien d'un troisième type de diffusion. Il existe là un tout indissociable qui suppose la possession du matériel comme condition nécessaire et suffisante $d u$ jeu. La règle n'est pas ce qu'apporte une communauté pour donner un sens à un matériel polyvalent, mais ce qui est inhérent au matériel même.

La transmission passe par l'achat d'un matériel qui livre avec lui ses règles. Non seulement les règles sont extérieures à la communauté des joueurs, mais aussi le matériel issu d'une fabrication industrielle. D'autre part, il n'est pas polyvalent et chaque matériel est lié à des règles spécifiques comme c'est le cas pour Monopoly (Miro) ${ }^{3}$ 
Richesses du Monde (Pachis, Nathan, 1969) ou l'Autoroute (Dujardin, 1968). L'acte même de jouer doit être précédé par la consommation d'un objet qui le rend possible.

Cette diffusion est liée à des techniques spécifiques; des techniques de fabrication: matériel homogène (fabrication en série) très semblable pour divers jeux (papier, texte, plastique); des techniques de diffusion: diffusion commerciale appuyée sur la publicité, une présentation sous la forme d'un coffret qui met en évidence le thème qui doit inciter à l'achat, rôle des grands éditeurs et de leurs réseaux. Le jeu existe là aussi, il ne s'agit pas de le nier, mais il est engendré par d'autres processus, en particulier la consommation d'un produit fini, complet et autosuffisant, avec lequel il est possible de jouer, le « prêt-à-jouer ». Ce qui différencie les divers produits offerts par le marché, ce n'est pas la valeur du jeu, mais l'attrait du thème. Le jeu entre ainsi dans l'ère industrielle par tous ses éléments. L'innovation n'est pas liée à une transmission de proche en proche à partir du foyer de création, ni à la diffusion de règles écrites, mais passe par la médiation d'un éditeur de jeu, intermédiaire nécessaire entre tout inventeur et l'ensemble des joueurs.

Tous ces détails peuvent paraître sibyllins, mais la situation des joueurs est ici toute autre ; ceux-ci se trouvent plongés dans une sphère de pure consommation de produits équivalents entre eux. Le jeu ne se déroule plus au seul niveau des joueurs, mais suppose l'intervention de la production et de la commercialisation. Loin que le jeu soit premier, il n'est plus que le résultat d'un acte banal de consommation (direct ou sous forme de cadeau). On pourrait parler de nouveaux rapports sociaux du jeu; non plus participation à une tradition, transmission collective de règles, mais acte d'un consommateur isolé : le jeu s'impose comme tout autre produit de consommation. Alors que dans la transmission écrite le jeu choisi devenait indifférent, ici il est devenu indifférent de faire l'achat d'un jeu, d'un jouet ou de tout autre objet de fonction équivalente (ayant pour but de meubler un temps de loisir). La communauté ne préexiste pas, mais devra se constituer après l'acte de consommation individuelle et sans nécessité aucune. Les relations de jeu n'ont plus le caractère de relations essentielles, mais peuvent se trouver réduites à une consommation en commun d'un nouveau produit. Le jeu est entré dans le domaine que nous avons décrit à propos du jouet industriel (Brougère, 1979).

Les différents niveaux analysés ne sont pas nécessairement des phases historiques, mais coexistent dans nos sociétés quel que soit par ailleurs le développement que prend le troisième type dont l'intérêt commercial est évident : chaque nouveau jeu implique un nouvel achat au lieu d'une utilisation différente d'un matériel unique. Toute transmission isolée de la règle est ici impossible. La règle est liée à la manipulation d'un matériel précis, et on ne peut l'isoler qu'abstraitement. C'est là par ailleurs la grande nouveauté de ces jeux qui permettent la manipulation d'entités quasi concrètes et non de purs signes comme le sont devenues les cartes ou les pièces d'un jeu d'échecs.

En effet, dans un jeu d'échecs les pièces n'ont de détermination que formelle d'après le type de déplacements qui les définit. Elles pourraient être toutes autres que celles que la tradition nous a transmises. Il en est de même pour le jeu de l'Oie qui possède une détermination formelle stricte et invariable au-delà de la multiplicité des supports. Il s'agit de 63 cases dont un certain nombre conduisent à des opérations précises de la part des joueurs. Le reste n'est que fioritures, sans rôle dans le jeu. L'évolution des supports des jeux de l'Oie (D'Allemagne, 1951) relève d'une autre histoire que celle des jeux, celle des représentations ou des idéologies. Le tableau du jeu servit de support à 
des thèmes pédagogiques ou politiques. Mais si ce support est aussi facile à utiliser, c'est que les thèmes rapportés n'ont aucune incidence sur le jeu, sous condition que le formalisme soit respecté. Le jeu est indépendant du matériel sous réserve que celui-ci respecte les règles nécessaires.

$\mathrm{Au}$ contraire dans le jeu d'édition, le fait de jouer est solidaire d'un matériel précis. Le Monopoly ne se réduit pas à des déplacements formels sur un tablier, mais est aussi la manipulation d'argent, de cartes, d'objets divers, de tout un matériel déterminé sans lequel le jeu ne serait plus. Le changement de matériel implique un changement de jeu, même si les principes de base en sont les mêmes. La connaissance des règles d'un jeu de même type permet d'en comprendre le principe, mais non les opérations concrètes qu'il s'agit de réaliser dans ce jeu-ci. Dans la mesure où l'achat du jeu d'édition est ce qui rend possible le jeu même, il introduit un tout autre univers ludique. Les joueurs sont soumis à une autre instance, celle de la fabrication/diffusion qui leur livre la possibilité de jouer.

30 La multiplicité actuelle des jeux thématiques présentés aux acheteurs ne peut que rendre difficile l'appréciation de leurs possibilités réelles de jeu. Ils ne se différencient au premier abord, et rarement après analyse, que suivant le thème évoqué. Dans la mesure où chaque matériel ludique n'a qu'une seule fonction et n'est aucunement polyvalent, multiplier les jeux rend nécessaire la multiplication des achats de matériel, et donc redouble l'acte initial de consommation. Il n'est possible de renouveler la possibilité de jeu qu'en répétant l'acte et non pas en créant de nouvelles possibilités formelles sur un matériel préexistant.

31 Ce type de diffusion du jeu lui donne d'emblée un sens avant l'acte même de jouer : un sens non communautaire. Cette remarque a une portée plus large que ce qui nous occupe ici et regarde aussi bien le jouet acheté et, au-delà, de nombreux autres objets. Le mode de diffusion est aussi mode de présentation de l'objet et oriente le rapport qu'il aura avec l'utilisateur. Cet objet, ici le jeu, «vient d'ailleurs » dans tous les éléments qui le composent: par les thèmes qui font intervenir des mécanismes sans rapport avec l'expérience vécue du joueur, par sa fabrication (matériaux et technologies de la fabrication industrielle), par sa diffusion. Le joueur « doit faire » avec un objet extérieur, s'initier aux règles et à son aspect, tenter de se l'approprier à défaut de l'avoir reçu comme un «savoir-jouer », à défaut de l'avoir recréé.

Nous devons évaluer l'aspect novateur de ces jeux au-delà même de la nouveauté de leur contenu, dans un nouveau rapport social au jeu. Le jeu n'est plus l'affaire des joueurs, mais de spécialistes et de fabricants. Bien sûr, il faut reconnaître qu'ils interviennent là où les traditions, le savoir-jouer ont commencé à disparaître (rôle de la télévision, des automobiles et de nouvelles formes de loisirs) et en un sens compensent un manque, mais sur un tout autre mode. Il est évident que les transformations sociales et matérielles (habitat, isolement social dans la famille, rupture des communautés traditionnelles) ont rendu possibles ces jeux dont les premiers sont apparus aux ÉtatsUnis ${ }^{4}$. Ils ne doivent leur succès qu'à la disparition au moins partielle des anciens rapports au jeu. Le savoir des éditeurs s'est imposé sur le non-savoir des joueurs, sur la disparition du savoir-jouer. Ce mouvement est parallèle à celui qui, au sein du monde du jouet, voit l'industrie du jouet se développer à partir des traditions relatives soit au jeu, soit à la fabrication des jouets par les parents ou les enfants. La mutation technologique s'effectue sur le fond d'une mutation plus profonde qui touche le rapport que l'on entretient aux autres, à son histoire, à la nature. La rupture des 
transmissions traditionnelles (orales, pratiques d'imitation) rend possible la diffusion commerciale de ce qui naguère y échappait.

\section{Hasard et stratégie}

Avant de faire quelques remarques sur les règles formelles mises en œuvre par les jeux thématiques, il est nécessaire de rappeler brièvement les distinctions courantes touchant les règles des jeux de société. On distingue ainsi trois types de jeux : les jeux de pur hasard (roulette, jeu de l'Oie), les jeux de stratégie ou de combinaison pure, réglés exclusivement par la science des adversaires (échecs, dames, jeu de go, Awalé), enfin un mélange des deux, où l'habileté des joueurs consiste à tirer le meilleur parti des circonstances accidentelles (jeux de cartes, trictrac et dérivés comme le jacquet) ${ }^{5}$.

Les jeux de hasard pur dont le résultat est absolument indéterminé sur un coup (mais dont le calcul des probabilités donne les chances respectives des possibilités) est complètement déterminé pour le joueur dans la mesure où celui-ci ne peut que suivre, dans les opérations qu'il fait éventuellement, ce que le sort lui indique. Ainsi dans le jeu de l'Oie, la mobilité du pion est liée aux points indiqués par le dé et consécutivement aux cases sur lesquelles conduit cette indication. A l'opposé, le jeu d'échecs exclut le hasard. Il est à remarquer cependant que pour un joueur moyen qui ne peut déterminer, en analysant les conséquences de ses gestes, ce qu'il doit jouer, le hasard intervient. Quant aux jeux qui mélangent hasard et stratégie, ils sont différents selon que le hasard n'intervient qu'au début (jeux de cartes) et laisse pour le reste agir la science du joueur, ou selon qu'il intervient continuellement (trictrac), rendant impossible la construction d'une stratégie cohérente pour l'ensemble d'une partie.

Les jeux thématiques semblent avoir pour schéma de base celui du jeu de l'Oie, c'est-àdire le pur hasard; il intervient cependant selon plusieurs modalités : dés, opérations relatives à la position des pions et tirages de cartes dites "chances». Cependant, et c'est là leur originalité, il existe dans ces jeux d'autres opérations qui ne sont pas directement réductibles aux aspects formels de ces jeux, mais qui peuvent laisser une part, difficilement évaluable, à la stratégie : il s'agit d'opérations concrétisées comme celles d'achat ou de vente (on peut ainsi en partie choisir les moments d'achat, donc établir dans les limites du possible, selon ce que permet le hasard, des stratégies d'achat, qualitatives ou quantitatives). D'autres jeux (tel Monsieur le Président, Robert Laffont) excluent toute stratégie.

Donc ces jeux sont un mélange original de hasard et de stratégie : au niveau purement formel, leur modèle est le jeu de l'Oie, c'est-à-dire le pur hasard, mais au niveau déjà thématique d'opérations concrétisées, il apparaît une possibilité de stratégie. D'où cet autre caractère qui est la difficulté, à l'inverse des jeux classiques, d'isoler les règles formelles des opérations concrétisées, lesquelles sont généralement des opérations commerciales ou imaginées sur ce modèle; c'est ce qui fait, nous l'avons déjà noté, que le jeu est dépendant de son matériel et de sa thématique.

L'effet principal de l'intervention du hasard est la mise à égalité des joueurs : il y a équiprobabilité des chances face à la victoire et à la défaite. Les jeux thématiques ne mettent pas en face les uns des autres des joueurs ayant acquis par la pratique, comme aux échecs, des avantages différents, mais au contraire toujours égaux à chaque nouvelle partie. L'autre effet, dû à la faiblesse de la stratégie, est la similitude des parties successives et la nécessité de recourir à un autre jeu pour pouvoir introduire de 
la variété. Des parties qui se déroulent selon les injonctions du hasard ne varient que très peu les unes par rapport aux autres. Seule la stratégie permet de varier à l'infini les parties possibles.

Or, la seule façon de donner de l'intérêt au jeu de hasard est justement de "l'intéresser ", de le soumettre à un gain et à une perte. C'est le cas des pratiques courantes relatives au poker, aux divers jeux de dés. C'est aussi le cas, dans la règle originelle, du jeu de l'Oie qui fut longtemps un jeu de salon pour adultes. Ainsi, aux obstacles marqués par les cases correspondent des pénalités financières dont la totalité ajoutée à la mise de départ est emportée par le vainqueur de chaque partie. Le jeu de l'Oie apparaît comme un jeu d'argent qui permet cependant, mais pas toujours, de ne jouer que des sommes assez faibles. Aux XVI ${ }^{e}$ et XVII ${ }^{\mathrm{e}}$ siècles, les jeux d'argent n'étaient pas limités à des lieux spécifiques, mais répandus dans la société. De même les enfants et adolescents avaient la possibilité de jouer de l'argent quelles que fussent les tentatives de limitation par les pédagogues qui durent souvent, jusque dans les collèges, en entériner la pratique pour éviter de voir leurs élèves les plus âgés tenter leur chance dans les salles de la ville (Ariès, 1973). Jusqu'au XIXe siècle, les enfants n'étaient donc pas exclus des jeux d'argent.

Dans la mesure où peu à peu furent interdits effectivement aux enfants les jeux d'argent (qui peuvent se maintenir à travers les jeux de billes ou autres, qui confèrent aux vainqueurs l'acquisition d'objets à forte valeur symbolique), les jeux actuels d'édition apparaissent comme leurs succédanés. Mais l'argent n'est plus un élément extérieur au jeu, il y est intégré par imitation d'activités sociales qui utilisent l'argent ; la morale est sauve. Ces jeux ont ainsi permis de conserver l'argent comme intérêt émotif sans enfreindre une nouvelle conception des rapports de l'enfant à l'argent qui l'exclut de sa manipulation effective. Il est de fait que l'aspect réaliste des unités monétaires est particulièrement soigné dans ces jeux. Là où l'argent n'est pas utilisé, il existe des unités d'échange qui ont le même rôle (ainsi les voix d'électeurs dans Monsieur le Président). La partie ainsi « intéressée » peut se limiter au hasard.

Dans les jeux classiques, il y a une indépendance du formel (du syntaxique) par rapport à la sémantique, à la représentation concrète. Ainsi en est-il pour les échecs ou les jeux classiques de cartes. On peut même juger de leur intérêt à partir de leur analyse formelle. Il n'en est pas de même pour les jeux, où la description formelle classique ne peut que passer à côté de son intérêt spécifique. A côté de l'aspect abstrait (type jeu de l'Oie), il faut ajouter l'échange d'entités concrètes conformes à certaines lois. Le « concret » semble donc devoir prendre de l'importance par rapport à l'aspect formel des jeux classiques, comme si les enfants étaient devenus incapables de jouer avec des entités abstraites.

41 Les jeux thématiques possèdent donc deux niveaux de règle : un classique, à l'imitation du jeu de l'Oie; des règles d'échange qui doivent se concrétiser en opérations financières ou toute autre activité sociale. La variation, l'innovation dans ces jeux se situent dans la traduction différente donnée aux règles d'échange, et parfois par quelques variations sur ces règles elles-mêmes, sans toucher aux principes qui demeurent les mêmes. Le premier niveau conditionne le second dans la mesure où les règles d'échange sont subordonnées au hasard (accès à telle case, tel chiffre, tirage de cartes). Mais si celles-ci sont déterminées quant au moment et lieu de leur intervention, elles sont indépendantes dans leur mécanisme ; elles fixent un objectif (réaliser, comme c'est souvent le cas, un monopole) et des moyens pour y parvenir. Les possibilités 
stratégiques, tout entières réduites à ce deuxième niveau, sont cependant limitées d'une part par l'intervention constante du hasard, d'autre part par le choix limité d'opérations différentes qu'il est possible de réaliser (accumulation monétaire, échange). Cet aspect stratégique a trop peu d'indépendance pour pouvoir donner un caractère de jeu de combinaisons réel à ces jeux.

Les opérations sociales en question ne sont pas reproduites par abstraction et formalisation, mais le plus fidèlement possible. La prégnance du hasard invalide d'autre part la fidélité de l'imitation par rapport à la réalité. Ce qui fait que ces jeux restent des jeux, c'est que les opérations demeurent soumises au hasard qui rend égales les chances des joueurs. Les échanges monétaires relèvent plus d'un ajout au jeu de hasard que d'une véritable reproduction de la réalité là où elle fait intervenir des stratégies concrètes. Pour nous résumer, le modèle est donc bien le jeu de l'Oie, mais complet, c'est-à-dire intéressé par l'engagement de sommes d'argent.

De ces deux niveaux formels que nous avons mis en évidence, il en est un qui est la traduction dans le jeu de certaines activités sociales qui prennent la forme d'échanges entre agents économiques. Le contenu thématique du jeu est lié à cette nécessité de faire appel à des données qui relèvent de ce type d'attitude, sinon réellement, tout au moins dans la façon dont on peut se la représenter. Cet appel à des contenus précis est ce qui marque fortement l'aspect du jeu. En ce sens, ces jeux d'édition ne sont pas seulement des jeux, mais ils sont signifiants avant même d'avoir pris un sens par le fait d'être joués, contrairement aux jeux traditionnels où seule la pratique confère un sens à des règles abstraites.

Avant de tenter de saisir le sens social de ces jeux, il est nécessaire d'analyser ce qu'ils représentent de la société, quels signes y sont effectivement manipulés. Ils sont caractérisés par la nécessité de jouer avec des éléments concrets déterminés, ce qui n'est pas le cas des jeux classiques où les éléments restent en grande partie abstraits. A des représentations effectivement abstraites (comme le tableau, parfois des pions), ces jeux ajoutent des éléments concrets: représentation spatiale du tableau, pions concrétisés (petites voitures du jeu l'Autoroute), une monnaie d'échange, des titres à négocier, éventuellement un matériel annexe sans valeur directe dans le jeu, ayant pour but de matérialiser plus précisément la thématique (téléscripteur de Envoyé spécial, Milton Bradley, 1976).

Figure 1

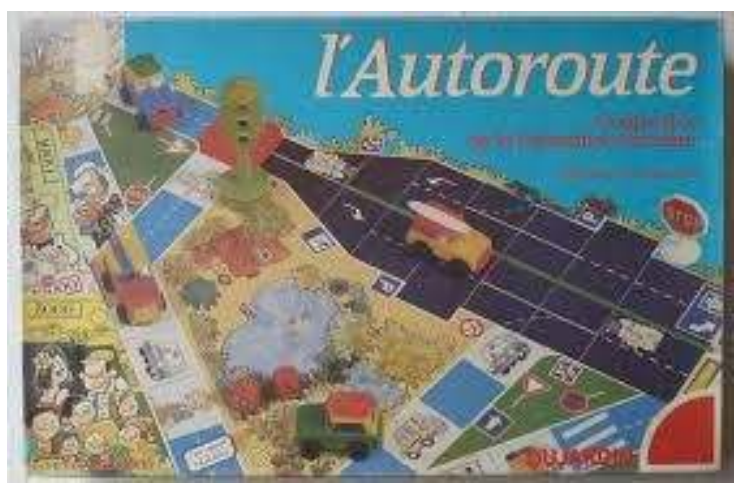

L'autoroute, Dujardin, 1968 


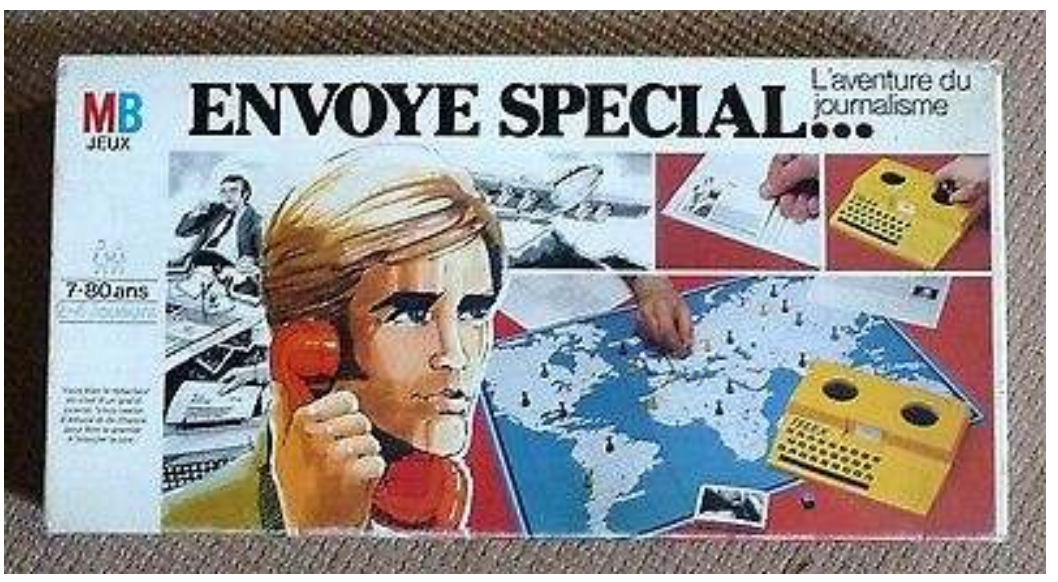

Envoyé spécial, Milton Bradley, 1976

La nécessité de se plier à certaines règles d'échange entre joueurs détermine les thèmes évoqués qui pour la plupart renvoient au domaine des échanges économiques pour s'élargir par analogie à d'autres mécanismes sociaux :

- échanges économiques : promotion immobilière (Monopoly), vente (Marketing, Nathan, 1974), Bourse (Jeu de la Bourse, Maier, Ravensburger, 1972), matières premières et échanges économiques internationaux (Pétropolis, de Rosnay, Nathan, 1976) ; Long cours, Miro, 19596; Richesses du Monde), achat d'animaux (Wild Life, Ravensburger, 1964);

- autres thèmes sur ce même modèle: élections où la voix est monnaie d'échange et la circonscription, titre négociable (Monsieur le Président), la conception d'un journal où l'information est le titre négocié (Envoyé spécial).

La matrice de départ est l'échange économique où la stratégie doit permettre aux joueurs d'accumuler le maximum de biens de façon à réaliser un monopole et réduire les autres à la "faillite ». Les partenaires égaux, c'est-à-dire mis au départ dans des conditions égales, doivent, selon un cheminement soumis au hasard, accumuler les titres négociables. Le gagnant, à défaut d'avoir réduit totalement ses adversaires, est celui qui a accumulé le plus de titres aux moindres frais. Il suffit pour créer un nouveau jeu d'appliquer ce schéma à un autre domaine de la réalité, soumis, de préférence, à l'intérêt des acheteurs potentiels par l'actualité (voir les jeux autour du thème du pétrole).

Pour s'intégrer au jeu, la réalité préexistante doit être au préalable pensée dans les termes du modèle du jeu, c'est-à-dire que les éléments concrets manipulés par le joueur sont en fait le résultat d'une abstraction et d'une interprétation de la réalité à travers le jeu. Il y a effectivement représentation matérielle d'un espace, d'éléments de la réalité sociale. Mais ce ne sont que des figures, des représentations abstraites (séparées) de la réalité, inscrite uniquement dans leur mode de présentation. A travers cette volonté de concrétisation on n'obtient que la représentation d'un monde abstrait et vide car dénué de signification autre que celle d'un espace qui, comme le jeu de Go, à travers un espace abstrait donne le modèle d'un rapport possible à l'espace concret. Ce qui, par le jeu, est concrétisation de ces éléments, est au regard de la réalité abstraction des données. Dans la mesure où ils sont nécessairement régis par des règles, les jeux d'édition sont tout aussi abstraits que les jeux classiques. Ils ne font que déjouer cette abstraction par 
la présence de signes manipulés qui se veulent réalistes ou tout au moins évocateurs de la réalité.

Il en est de même pour l'unité d'échange employée : ce qui importe c'est le rôle qu'elle a dans le jeu en en faisant un échange généralisé. L'aspect concret dans sa représentation ne change en rien son usage selon des règles précises. Le jeu d'édition ne fait que déterminer par le renvoi à une réalité existante, ce qui n'a pas besoin de l'être dans le jeu. On ne peut juger de l'aspect concret d'un jeu aux signes qu'il utilise mais à son adéquation vis-à-vis d'une réalité sociale. Cette volonté de mimer au plus près les éléments de la réalité n'a d'intérêt que commercial, dans le but de séduire le joueur. Pour le jeu, la nouveauté est d'avoir admis, en plus du hasard, des règles permettant d'appliquer au jeu de tels contenus précis. La finesse d'imitation est secondaire quant aux possibilités du jeu. La recherche de cette précision relève d'une autre fonction de ces jeux, celle qui consiste à permettre une identification du joueur au rôle qu'on lui propose.

Nous arrivons donc à une conclusion contradictoire : le contenu de ces jeux est à la fois rendu nécessaire et inessentiel pour les règles formelles: il est nécessaire que le deuxième niveau formel (celui qui ne relève pas du jeu de hasard) s'exprime à travers un contenu qui indique ce qu'il faut échanger et contre quoi, mais tout contenu qui peut adopter cette forme peut être utilisé. La production du jeu, ne conduit pas à réaliser un jeu à partir d'une activité sociale, mais transforme les données sociales de façon à les adapter au principe du jeu. Il en résulte ceci : loin que le jeu puisse alors servir pour la compréhension de la réalité, c'est l'existence préalable des règles du jeu qui implique la forme donnée à la réalité pour être intégrée au jeu. De la réalité sociale n'est retenu que ce qui est conforme au schéma indispensable au jeu: ainsi de la constitution d'un zoo ne sont retenus que l'achat, la vente des animaux (Wild Life); ou d'une élection : le comptage des voix et des circonscriptions. Il suffit que cette réalité, par un de ses aspects, aussi minime soit-il, puisse s'adapter au schéma formel, pour qu'il forme le contenu possible du jeu. Longue, bien entendu, est la liste des aspects qui échappent nécessairement à ces types de jeux. Ce qui est retenu de la réalité est ce qui peut rendre possible une stratégie en termes de maximum des échanges entre partenaires au départ égaux.

Le choix de simplification n'est pas idéologique au premier titre - même si elle peut avoir un effet idéologique - mais lié aux contraintes mêmes du jeu, et à leur acceptation. Moins qu'une tentative de reproduire la société par des jeux, il s'agit plutôt de la société mise en jeu, adaptée à un jeu préexistant. Dans ces conditions on voit mal la valeur heuristique ou pédagogique de tels jeux. Cependant le schéma formel de ces jeux, s'il ne présente qu'un aspect de la réalité, n'en présente pas moins un aspect effectif par ces jeux, tout au moins pour certains. En cela le jeu purement ludique ici rencontre les analyses économiques qui utilisent le jeu pour comprendre sinon toute la réalité sociale du moins certains de ces aspects. C'est sur cette similitude que nous allons, maintenant, nous interroger.

\section{Simulation, théorie et jeux}

51 Dans ce qui précède, nous n'avons analysé que les structures qui permettent le jeu et non pas le sens du jeu. Pour celui-ci, dans son aspect général, nous ne pouvons proposer que des hypothèses. La question est de savoir en effet que signifie, dans une 
société donnée, de jouer à partir de règles déterminées. Pour pouvoir comprendre les jeux d'édition de ce point de vue aussi, nous allons partir, à titre d'hypothèse, des propositions de Marshall McLuhan. Selon lui, les jeux, émanation de l'art populaire, sont les représentations des actions quotidiennes de l'homme dans une société donnée, les modèles d'une culture. Ils permettent d'atteindre des situations moins accessibles d'une autre façon: «Les jeux sont des situations inventées pour permettre la participation simultanée de plusieurs personnes à des modèles importants de leur vie collective. » (McLuhan, 1977, p. 269)

Dans le jeu, l'homme aurait ainsi le modèle du fonctionnement de sa propre société, ou du moins de certains de ses aspects ${ }^{7}$. Mais si ceci peut paraître vrai dans certaines sociétés traditionnelles (et c'est encore à vérifier) qu'en est-il dans nos sociétés ? Ce qui doit nous frapper c'est que la valeur de modèle du jeu est reconnue comme telle depuis au moins plusieurs dizaines d'années par le développement de la théorie des jeux. L'ouvrage d'Oscar Morgenstein et John von Neuman, Theory of Games and Economic Behaviour, date de 1944. Sans vouloir présenter l'objet de cette théorie, nous pouvons au moins rapidement résumer quelle utilisation elle fait du jeu. Elle part du fait qu'il est possible de mathématiser les jeux, qu'il existe une théorie mathématique des jeux, aussi bien des jeux de stratégie que des jeux de pur hasard. Or les jeux sont une suite d'actions humaines réglées par des conventions respectées et une suite de décisions prises en fonction d'un objectif précis. Il suffit donc de décrire une situation économique (ou autre selon les développements ultérieurs) dans les termes d'un jeu pour pouvoir y appliquer une analyse mathématique. Penser la réalité, ou plus exactement certains aspects de la réalité où la décision humaine, la stratégie interviennent, sous la forme de jeux, permet sa description mathématique. En effet, cette théorie propose plus des descriptions de situations que des lois qui permettraient de déboucher sur des prévisions.

L'intérêt de la théorie des jeux réside dans la rigueur de la description, et cette rigueur est obtenue par la transcription de la réalité dans un modèle qui est un «jeu », dans la mesure où il ne concerne qu'une situation où existent joueurs, règles, stratégies possibles et information disponible. Le jeu apparaît ainsi comme un modèle simplifié d'une situation où la décision d'un agent doit intervenir. Cela tend à confirmer l'hypothèse dont nous étions partis.

Les modèles mis au point par la théorie des jeux ont deux usages pratiques majeurs : la simulation et l'emploi pédagogique. Dans la simulation, il s'agit d'appliquer au modèle construit toutes les stratégies que l'on est susceptible d'employer pour voir les différentes réactions possibles du modèle, en somme de jouer différentes parties et lire les résultats obtenus par les différents joueurs. Les jeux d'entreprise sont une application pédagogique du même principe. Il s'agit de faire jouer à des personnes qui auront des rôles précis à remplir dans leur activité professionnelle ce rôle à l'intérieur d'un jeu qui est le modèle du type de situation à laquelle ils seront confrontés :

A l'aide d'un modèle mathématique ont été reconstituées les principales relations de cause à effet qui interviennent dans un ensemble de compagnies en compétition sur divers marchés ; ce modèle a été codé et programmé sur un ordinateur puissant. Les quatre équipes, composées de personnes compétentes, gèrent des entreprises fictives et prennent, phase par phase, les décisions qui concourent à la vie de ces firmes imaginaires. Le temps a été contracté; ce qui se passe ici en un jour se déroulerait en un an ou deux dans la réalité. Il s'agit en fait d'un jeu éducatif, d'une expérience au cours de laquelle ces personnages prennent passion et intérêt pour 
des situations artificielles. Ce n'est plus le simple jeu de Monopoly, qui amuse encore les enfants; les relations qui sont à la base des faits économiques et de la concurrence ont été figurées aussi bien que possible: tel est le jeu des entreprises. (Kaufman et al., 1964, p. 4-5). qu'une différence de degré (aussi importante soit-elle) et non une différence de nature. Dans les deux cas, la réalité est adaptée (pour des raisons purement ludiques, descriptives ou pédagogiques) à un schéma formel du jeu. La différence des projets et des résultats ne doit pas cacher l'identité des opérations. Pas plus le jeu d'entreprise que les jeux d'édition ne présentent tous les aspects de la réalité ; ils ne saisissent, par le moyen du principe de jeu, que certains aspects.

Mais la grande différence se situe dans les joueurs eux-mêmes. Alors que, dans les jeux d'entreprise, il y a correspondance entre le rôle des joueurs et leur fonction sociale réelle, pour les joueurs des jeux thématiques, il y a hiatus entre les situations qu'ils simulent dans le jeu, et, dans la plupart des cas, leur situation réelle. Ce qui est donc simulé par le joueur, ce n'est pas un rôle qui sera sien; ce n'est pas le préalable à une maîtrise ultérieure qui requiert un apprentissage, mais une maîtrise qui demeure constamment dans l'illusion. Tout au moins, il s'agit de cela dans les jeux effectivement proposés aux acheteurs, car rien n'interdit d'imaginer un jeu qui proposerait au joueur une situation en rapport avec sa situation réelle. Or l'existence de ce hiatus n'est pas liée uniquement aux jeux, mais au fonctionnement même de nos sociétés où la majorité est écartée des postes de maîtrise réelle des mécanismes. Nos sociétés ne sont pas faites de joueurs égaux. Or justement, c'est ce qui les distingue, en partie, des sociétés traditionnelles où il n'existe pas ce hiatus entre le joueur et l'individu social. Ce qu'il joue dans le jeu, face à un autre, n'est pas en rupture par rapport à une situation réelle ; le jeu peut donc avoir un rôle de simulation efficace des mécanismes sociaux.

Alors même que nos sociétés ont mis en évidence la valeur descriptive du jeu, les nouveaux jeux qu'elles créent perdent leur aspect de simulation effective qui ne se retrouve plus que dans l'utilisation des simulations sous la forme de jeux d'entreprise, dans la formation des cadres. Si l'on s'en tient à l'apparence, les jeux thématiques, dont certains sont assez sophistiqués pour prétendre à une reproduction fidèle des mécanismes économiques, sont semblables aux utilisations scientifiques et techniques du jeu. Mais dans le fait même du jeu, il y a une différence qui est celle des utilisateurs. On ne semble proposer aux acheteurs que de s'identifier à des situations qui leur échappent dans leur vie quotidienne.

Nous avions vu que la transmission commercialisée construisait une rupture entre le jeu et le groupe des joueurs. Le contenu de ces jeux est tout aussi extérieur au groupe des joueurs, et cela explique qu'ils ne puissent varier que de l'extérieur, par diffusion commerciale. Ce que ces jeux transcrivent n'est le plus souvent que la situation des diffuseurs, des fabricants plutôt que celle des joueurs. Ce type de diffusion ne peut qu'orienter en ce sens le choix des contenus. L'extériorité des situations présentées dans le jeu renforce l'extériorité de la transmission du jeu. Ils ne sont plus l'émanation $\mathrm{du}$ groupe, condition nécessaire pour qu'ils traduisent dans le jeu la situation des joueurs. Au niveau du groupe, ils deviennent vides de sens, et ne produisent qu'une illusion de maîtrise sans conséquence. Mais au niveau de la société qui les rend possibles, ils sont expressifs du rôle du jeu dans la description de certaines situations. 

majeure partie des cas, inadéquat à la situation effective du joueur. Il ne fait que représenter quelque chose d'extérieur à la vie même du joueur. Cette inadéquation, permettant identification, est sans doute la cause du succès de ces jeux. La différence de ces jeux avec les jeux classiques est que ces derniers pourraient être remplis d'un sens différent en fonction de la situation des joueurs potentiels. Jouer n'est plus reproduire des sens prédéterminés, mais créer une signification à une action de jouer même si elle n'est pas explicitée concrètement. Qui n'a vu un jeune enfant interpréter les épisodes de jeux simples tels que les Petits Chevaux ou le jeu l'Oie en termes de relations avec les personnes qui l'entourent? Un jeu trop chargé de significations élimine plus (à savoir tous les sens possibles à créer) qu'il n'apporte. les pratiques, ludiques courantes, il ne semble plus avoir la possibilité d'offrir un rôle semblable au niveau des communautés des joueurs. Ce qui s'efface, c'est la possibilité des différences que créent des communautés face à leurs jeux. Et la variété que proposent les jeux d'édition est largement illusoire puisque les principes sont toujours semblables. Alors même que la théorie des jeux permet de concevoir des jeux relatifs aux situations spécifiques, les jeux d'édition continuent à proposer des thèmes qui ne concernent pas directement les joueurs.

61 Ces jeux étudiés sont amplement significatifs de notre société, de la place qu'elle peut laisser au jeu devenu produit à consommer. Nous avons pu souligner comment le produit offert était extérieur, tant dans son contenu que dans son modèle de diffusion, aux joueurs auxquels il prétend s'imposer. Bien sûr, cette coupure est corrélative de la coupure qui existe dans nos sociétés entre ceux qui consomment et ceux qui produisent, ou plus exactement, puisqu'il s'agit parfois des mêmes personnes, entre les actes de consommation et les actes de production, tout aussi parcellisés les uns que les autres. Et le jeu, sous ses formes modernes, n'est pas issu du groupe des joueurs.

\section{BIBLIOGRAPHIE}

ARIES, P. (1973) L'enfant et la vie familiale sous l'ancien régime, Paris, Le Seuil

BROUGERE, G. (1979) « Du jouet industriel au jouet rationalisé » in R. Jaulin (dir.) Jeux et jouets. Essai d'ethnotechnologie, Paris, Aubier

BROUGERE, G. (1995) Jeu et éducation, Paris, L'Harmattan

BROUGERE G. et FRANÇOIS S. (dir.) (2018) L'enfance en conception(s). Comment les industries culturelles s'adressent-elles aux enfants? Bruxelles, Peter Lang

D'ALLEMAGNE R. (1951) Le noble jeu de l'oie, en France de 1640 à 1950, Grund, Paris

DENEUIL P.-N. (1979) « Jeux de société » in R. Jaulin (dir.) Jeux et jouets. Essai d'ethnotechnologie, Paris, Aubier

HABERMAS J. (1973) La technique et la science comme « idéologie », Paris, Gallimard 
KAUFMAN A., FAURE R. et LE GARFF A. (1964) Les Jeux d'entreprise, $2^{\mathrm{e}}$ ed., Paris, PUF, Que sais-je ? $\mathrm{n}^{\circ} 892$

JAULIN, R. (1977) Les Chemins du vide, Paris, Christian Bourgois

JAULIN, R. (dir.) (1979) Jeux et jouets. Essai d'ethnotechnologie, Paris, Aubier

DE LA MARINIERE (1665) Maison des jeux académiques contenant un recueil général de tous les jeux divertissant pour se réjouir et passer le temps agréablement, Lyon, Etienne Loyson

MCLUHAN M. (1977) Pour comprendre les médias, Paris, Mame/Le Seuil

MORGENSTEIN O. et VON NEUMAN J. (1944) Theory of Games and Economic Behaviour, Princeton University Press

POPOVA A. et DELEDICK A. (1977) « Wari et Solo » : Le jeu de calcul africain, Paris, éditions CEDIC.

\section{NOTES}

1. Il s'agit des jeux à règle et non des jeux spontanés.

2. Note de 2021 : Le titre complet en est Maison des jeux académiques contenant un recueil général de tous les jeux divertissant pour se réjouir et passer le temps agréablement et la première édition, sous un autre titre, semble daté de 1654 .

3. Note de 2021 : L'éditeur indiqué est celui qui éditait les éditions françaises des jeux cités en 1978. Ainsi, Miro est une société qui a disparu à la suite de diverses fusions. Nous n'indiquons pas de date pour Monopoly dans la mesure où cela renvoie à une interprétation concernant l'origine. Je renvoie au texte de Bruce Whitehill (dans ce numéro). Pour les autres jeux la date, l'éditeur et l'auteur n'ont été indiqués que s'ils étaient présents dans le texte de 1979 ou s'il a été possible de les retrouver.

4. Note de 2021 : Voir dans le même numéro 14 de Sciences du jeu l'article de Bruce Whitehill

5. Note de 2021 : Je reprenais ici la façon dont l'Encyclopédie de Diderot et D'Alembert analysait le jeu, ce que j'ai développé dans Brougère, 1995, pp. 56-60

6. Note de 2021 : L'édition de référence au moment de cette recherche est postérieure (réédition des années 1970). Par ailleurs le jeu a été conçu et édité avant cette date :

https://fr.wikipedia.org/wiki/Long_Cours_(jeu_de_soci \%C3 \%A9t \%C3 \%A9). Cela peut être vrai d'autres jeux cités.

7. On peut se reporter à ce sujet aux analyses des rapports entre un jeu, le mankala, et les mécanismes sociaux dans Popova et Deledick, 1977, p. 42 et sqq.

\section{RÉSUMÉS}

Précédée d'une présentation originale, cet article est la réédition d'un texte publié en 1979. Il explore la spécificité des jeux d'édition dont la diffusion des règles est solidaire de la commercialisation du matériel. Il s'agit d'une nouvelle modalité de construction d'une culture ludique après la transmission orale ou par observation et l'usage de livres de règle. Ce nouveau cadre de jeu implique un matériel original et non l'usage d'un matériel polyvalent comme les jeux traditionnels de cartes ainsi que l'absence d'une communauté préexistante de joueurs. Les 
jeux thématiques associant hasard et échanges monétaires fictifs, très présents dans les années 1970, sont plus particulièrement étudiés. Le texte se termine par une comparaison de ces jeux à la théorie des jeux et aux jeux d'entreprise, en soulignant le travail de modélisation et de simplification que l'on trouve dans ces divers dispositifs.

Preceded by an original presentation, this article is a re-edition of a text published in 1979. It explores the specificity of edition games, whose rules are linked to the commercialization of the material. It is a new way of constructing a ludic culture after the oral transmission or by observation and the use of rule books. This new framework for games involves original material and not the use of polyvalent material such as traditional card games, as well as the absence of a pre-existing community of players. The thematic games associating chance and fictitious monetary exchanges, which were very present in the 1970s, are more particularly examined. The text ends with a comparison of these games with the theory of games and business games, highlighting the work of modelling and simplification that can be found in these various devices.

\section{INDEX}

Mots-clés : jeu de société, transmission des règles, culture matérielle, communautés

Keywords : board game, transmission of rule, material culture, communities

\section{AUTEUR}

\section{GILLES BROUGÈRE}

Experice, Université Sorbonne Paris Nord 